\title{
Application of Ultrasonic Bone Curette in Endoscopic Endonasal Skull Base Surgery: Technical Note
}

\author{
Milton M. Rastelli, Jr. ${ }^{1}$ Carlos D. Pinheiro-Neto ${ }^{2}$ Juan C. Fernandez-Miranda ${ }^{1}$ Eric W. Wang ${ }^{2}$ \\ Carl H. Snyderman ${ }^{1,2}$ Paul A. Gardner ${ }^{1}$
}

${ }^{1}$ Department of Neurological Surgery, University of Pittsburgh School of Medicine, Pittsburgh, Pennsylvania, United States

${ }^{2}$ Department of Otolaryngology, University of Pittsburgh School of

Address for correspondence Juan C. Fernandez-Miranda, MD, Department of Neurological Surgery, University of Pittsburgh Medical Center, 200 Lothrop Street, Suite 500, Pittsburgh, PA 15213, United Medicine, Pittsburgh, Pennsylvania, United States

\begin{abstract}
Background Endoscopic endonasal surgery (EES) of the skull base often requires extensive bone work in proximity to critical neurovascular structures.

Objective To demonstrate the application of an ultrasonic bone curette during EES.

Methods Ten patients with skull base lesions underwent EES from September 2011 to April 2012 at the University of Pittsburgh Medical Center. Most of the bone work was done with high-speed drill and rongeurs. The ultrasonic curette was used to remove specific structures.

Results All the patients were submitted to fully endoscopic endonasal procedures and had critical bony structures removed with the ultrasonic bone curette. Two patients with degenerative spine diseases underwent odontoid process removal. Five patients with clival and petroclival tumors underwent posterior clinoid removal. Two patients with anterior fossa tumors underwent crista galli removal. One patient underwent unilateral

\section{Keywords}

- endoscopic endonasal

- skull base

- bone removal

- ultrasonic bone curette optic nerve decompression. No mechanical or heat injury resulted from the ultrasonic curette. The surrounding neurovascular structures and soft tissue were preserved in all cases.

Conclusion In selected EES, the ultrasonic bone curette was successfully used to remove loose pieces of bone in narrow corridors, adjacent to neurovascular structures, and it has advantages to high-speed drills in these specific situations.
\end{abstract}

\section{Introduction}

Over the last decade we have witnessed the rapid evolution of endoscopic endonasal surgery (EES) with advancements in surgical techniques, equipment, and instruments. ${ }^{1-3}$ Technological advancements continue to drive the evolution of EES with the application of new technologies from other surgical disciplines. The benefits of new technologies include improved operative efficiency and less surgical morbidity.
A key tenet of skull base surgery is maximal bone removal to increase surgical access and minimize neurovascular manipulation. Drilling of certain bony prominences in the skull base, such as the anterior clinoid, entails risk for damage to adjacent neurovascular structures, such as the optic nerve or internal carotid artery (ICA). The ultrasonic bone curette was first introduced in skull base surgery to minimize this risk, and its application in EES was reported ${ }^{4-7}$ However, these previous studies have provided little information about the received

May 5, 2013

accepted

July 1, 2013

published online

February 17, 2014 (c) 2014 Georg Thieme Verlag KG Stuttgart · New York
DOI http://dx.doi.org/ 10.1055/s-0033-1354580. ISSN 2193-6331. 
usefulness of this surgical tool for endonasal skull base approaches, and applications have been limited to sellar and suprasellar regions. In this article, we describe the technical nuances, surgical applications, limitations, and advantages provided by the use of the ultrasonic bone curette in endoscopic skull base surgery. We demonstrate that this surgical device allows for safe and effective bone removal in critical areas of the skull base and enhances safety when working in narrow surgical corridors surrounded by dura and/or important neurovascular structures.

\section{Methods}

Ten patients (three men and seven women), mean age 51.7 (18-82) years, with skull base lesions underwent EES between September 2011 and April 2012 at the University of Pittsburgh Medical Center (Pittsburgh, PA, USA). High-speed drill and bone rongeurs were used in all cases for most of the bone work during the exposure. The ultrasonic bone curette (Sonopet Ultrasonic Aspirator, Stryker Corporation, Kalamazoo, MI, USA) was used for the precise removal of specific bony structures in critical areas, usually adjacent to neurovascular structures.

The Sonopet Ultrasonic Aspirator unit has a main console, a pedal, cables, and tubing to connect the handpiece. The suction and irrigation pumps are built into the main unit, which is out of the surgical field. The device removes tissue using energy from ultrasonic frequency vibration created by a piezoelectric element delivered to the handpiece. There is a universal handpiece $12.9 \mathrm{~cm}$ in length and $2.2 \mathrm{~mm}$ in diameter. An extended curved tip has been created for use endonasally, the Superlong Endoscopic Straight Tip, which is $20.15 \mathrm{~cm}$ in length and $1.5 \mathrm{~mm}$ in diameter (-Fig. 1A). Different sizes and angles for the cutting surface are available, which allows side action without damaging tissue adjacent to the opposing smooth surface: the Superlong Payner $360^{\circ}$, $19.58 \mathrm{~cm}$ in length, and the Superlong Open Angle Micro Claw and Superlong Micro Claw, both $19.59 \mathrm{~cm}$ in length (-Fig. 1B). The Micro Claws have a limited cutting surface, which means just one side of this tip is the active cutting zone (-Fig. 1C).

The tip combines the oscillation of longitudinal and torsional vibrations. Longitudinal excursion distance is minimal at $\sim 14 \mu \mathrm{m}$, which results in a predominantly torsional movement. The movements occur in $25 \mathrm{KHz}$ frequency and $0.36 \mathrm{~mm}$ width amplitude. There is also another, less aggressive handpiece specific for soft tissue debridement with a 34$\mathrm{KHz}$ frequency. Because of the nonrotational design, soft tissue and cottonoid pledgets are not grabbed and spun by the tip, ${ }^{7-9}$ and there is little or no torque, both potentially negative features of a standard drill.

Minimal pressure is necessary during the bone drilling because a light touch of the tip promotes bone fragmentation. Simultaneously, the equipment uses irrigation directly through the tip, offering continuous cooling and thereby avoiding heat injury to the nearby neurovascular structures. Finally, continuous aspiration via the tip removes the bone fragments and the water from the surgical field. The three

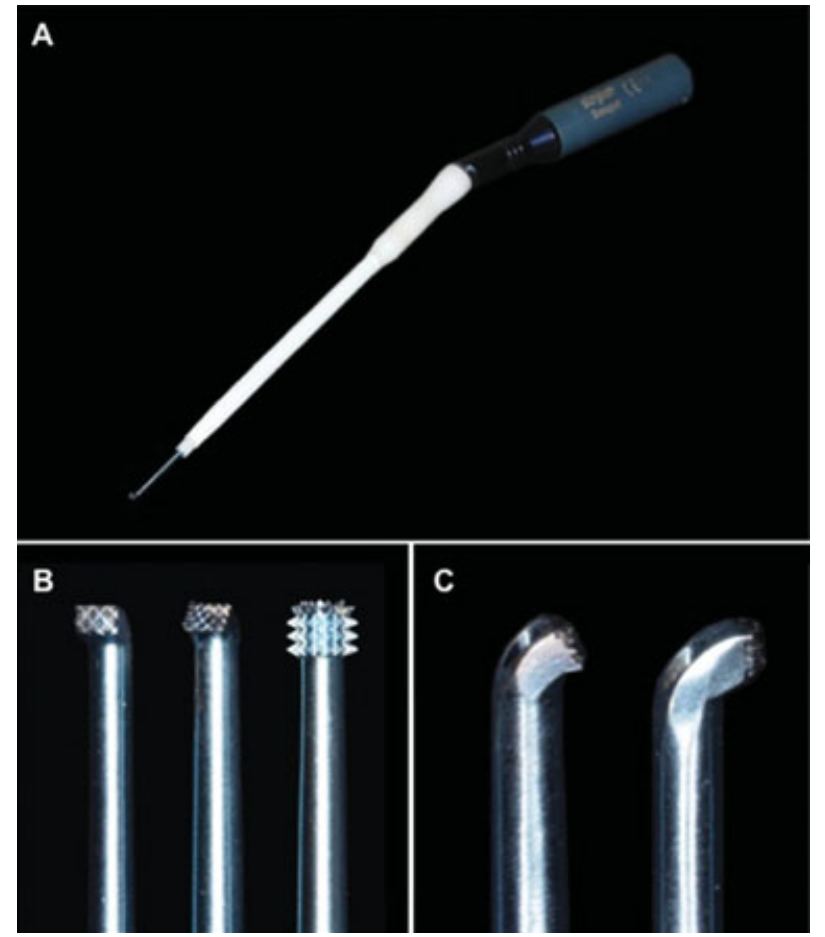

Fig. 1 (A) The extra-long handpiece of Sonopet for endoscopic endonasal surgery. (B) The three different tips used for bone removal (left to right): the Open Angle Claw, the Micro Claw, and the Payner $360^{\circ}$. (C) A lateral view to demonstrate the difference between the Micro (left) and the Open Angle (right), both with a one-side cutting surface.

variables (frequency of oscillation, irrigation, and aspiration) can be independently controlled and adjusted from the main console to provide the ideal performance for each case.

\section{Results}

One patient with osteoarthritis and one with os odontoideum (-Fig. 2A and 2C) underwent an endoscopic endonasal resection of the odontoid process due to irreducible symptomatic cervicomedullary compression. The ultrasonic bone curette was less efficient than the drill during the less delicate portions of bone removal such as the anterior ring of the atlas and the initial dens resection. However, in the final stages of dissection of the inner cortex, the aspirator was believed to be more controlled for bone removal in this critical area adjacent to the adherent tectorial membrane and ligaments (apical, alar, and transverse) (-Fig. 2B). With standard dissection techniques, a rim of adherent bone often remains at the most compressive portion of the tip of the odontoid. Safely dissecting this free-floating bone from the densely adherent ligaments can be challenging yet critical for complete decompression. The light touch of the ultrasonic curette allowed a controlled bone fragmentation of the odontoid process and a complete resection in both cases. Although this can be achieved using a drill and rongeurs, this new technique was believed to provide improved control in this deep, narrow corridor. In addition, the nonrotational mechanism 

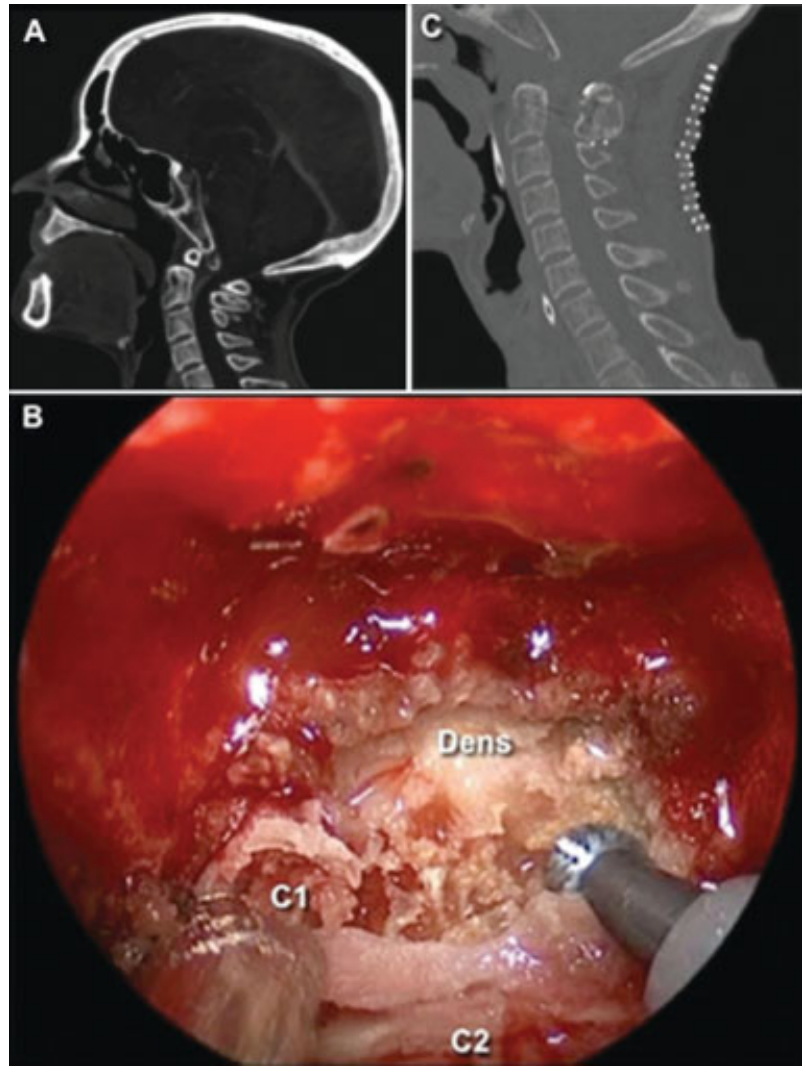

Fig. 2 (A) Preoperative sagittal computed tomography scan demonstrating the separation of the odontoid process from the body of the axis. (B) Extended endoscopic endonasal approach to resection of the odontoid process. The Payner $360^{\circ}$ is used after removing the anterior arch of $\mathrm{C} 1$. (C) Postoperative image demonstrating removal of the odontoid process.

avoids damage to the surrounding soft tissues that could occur when using extended high-speed drills.

Five other patients underwent transclival EES, three for a clival chordoma, one for a petroclival chondrosarcoma, and one for a petroclival meningioma ( $\mathbf{F i g .} \mathbf{3 A}$ and $\mathbf{3 C}$ ). In these cases, the ultrasonic curette was used for removal of the posterior clinoid (-Fig. 3B). The posterior clinoid is surrounded by dura and located deep in a narrow corridor bounded by the ICA (anterolateral), pituitary gland (anteromedial), and oculomotor nerve (posterolateral). The limited cutting surface of the ultrasonic curette permitted a safe and complete resection of the posterior clinoid process in all five cases, with no injury to the dura mater and surrounding neurovascular structures. Despite the directional selectivity of the ultrasonic curette, the ICA was gently retracted and protected with a suction tip whenever working in direct proximity. Similar to resection of the dens, if the posterior clinoid became detached from the dorsum sellae, controlled removal of the loose piece of bone was facilitated by the ultrasonic curette as detailed earlier.

Two patients had anterior cranial base lesions: a rightsided olfactory groove meningioma ( - Fig. $\mathbf{4 A}$ and $\mathbf{4 C}$ ) and an esthesioneuroblastoma involving bilateral olfactory clefts. The patient with the right-sided meningioma had a unilateral
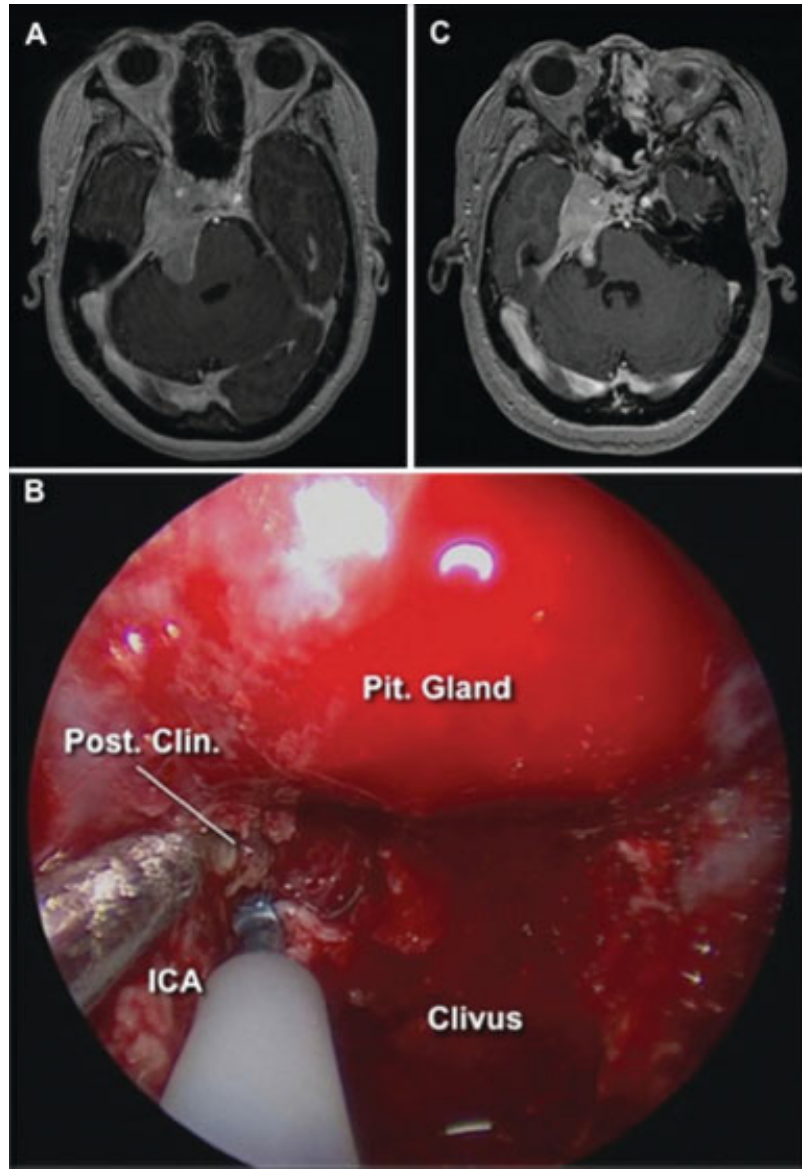

Fig. 3 (A) Preoperative axial magnetic resonance imaging scan demonstrating a right-sided petroclival meningioma compressing the brainstem. (B) Endoscopic endonasal transclival approach. The Micro Claw is used to remove the right posterior clinoid. (C) Postoperative image demonstrating partial resection of the lesion and recovery from brainstem compression. ICA, internal carotid artery; Pit. Gland, pituitary gland; Post. Clin., posterior clinoid).

anterior cranial base resection via one nostril for preservation of olfaction; the patient with the esthesioneuroblastoma had a bilateral anterior craniofacial resection. In both cases, most of the bone of the anterior cranial base was removed with a high-speed drill and bone rongeurs. The ultrasonic bone curette was used to drill the right half of the crista galli on the first case ( $\mathbf{- F i g}$. 4B) and to remove the crista galli completely on the second one. After the dissection of the olfactory sulcus dura from the crista galli, the ultrasonic curette was placed in this narrow space between the dura and the bone. Its one-sided cutting surface allowed the safe drilling of the crista galli while maintaining the integrity of the adjacent dura. When the crista galli is partially or totally detached from the frontal bone, further drilling, dissection, and removal of the mobile bone fragment becomes more difficult. In both cases, it was possible to perform the resection of the crista galli with no dural injury.

The last patient, who presented with slight visual field loss, mild proptosis, and an afferent pupillary defect secondary to fibrous dysplasia (- Fig. 5A and 5C), underwent an endoscopic endonasal left optic nerve decompression. In this particular 

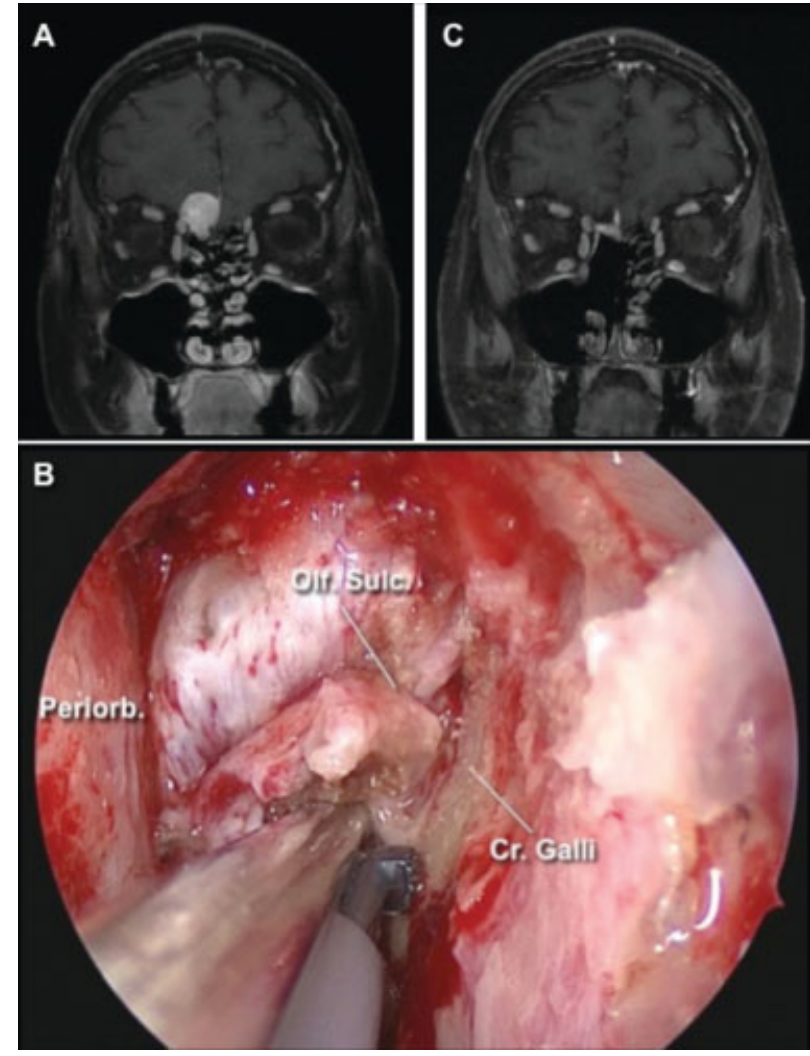

Fig. 4 (A) Preoperative coronal magnetic resonance imaging scan demonstrating a right-sided olfactory groove meningioma. (B) Endoscopic endonasal uninarial approach. The Micro Claw is used to remove the crista galli. (C) Postoperative image demonstrating gross total resection of the lesion. Cr, crista; Olf. Sulc., olfactory sulcus; Periorb., periorbita.

case, the optic nerve was displaced anteriorly and inferiorly by the tumor. The initial bone removal was performed with high-speed drills and rongeurs, including most of the roof of the optic canal. During the removal of the roof, a narrow corridor was created with the optic nerve immediately below. The ultrasonic bone curette was used to remove a small amount of bone over the optic nerve to achieve complete nerve decompression (-Fig. 5B). Again, its limited cutting surface permitted safe removal of bone from the roof of the optic canal without injury to the optic sheath.

In summary, the ultrasonic bone curette was effective in achieving the surgical goals in all 10 cases. No heat or mechanical injury was observed when the noncutting surface of the tip touched the dura or adjacent soft tissue. Nevertheless, structures such as the ICA were protected with a suction tip whenever the device was used in direct proximity. No acquisition of additional surgical skills was necessary to handle the ultrasonic curette.

\section{Discussion}

The use of ultrasonic devices is well established not only in neurosurgery but in many other medical specialties and also dentistry. ${ }^{9}$ For example, neurosurgeons have described its application to perform anterior clinoid removal
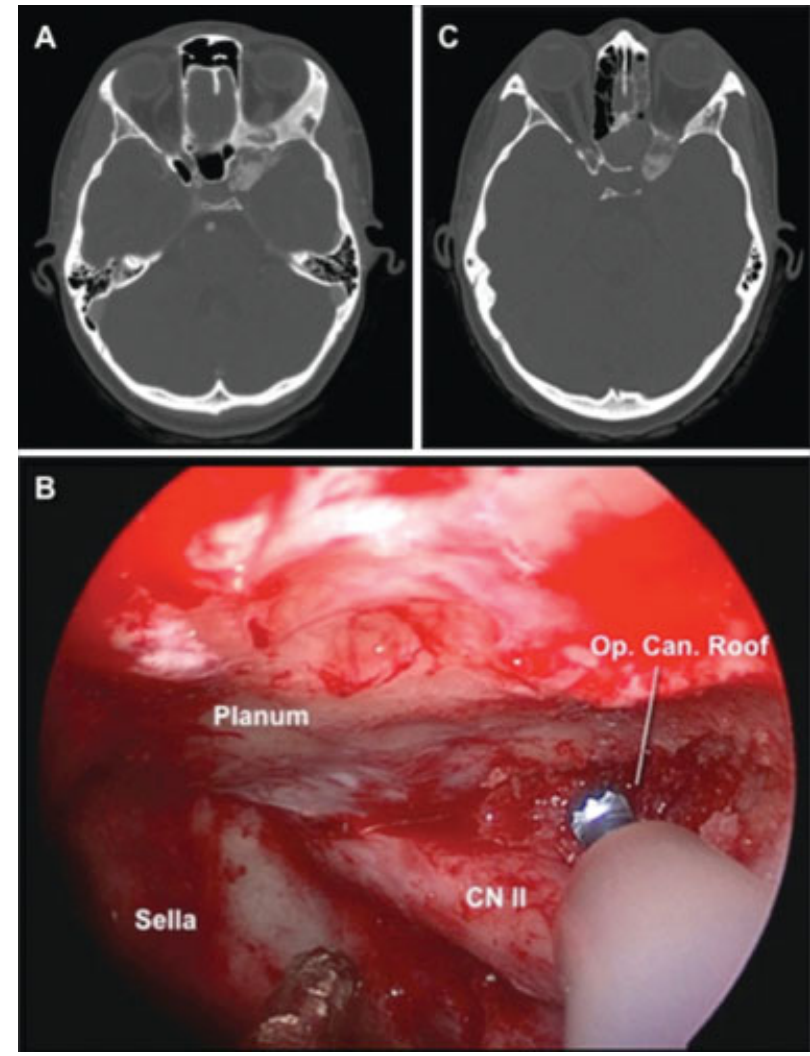

Fig. 5 (A) Preoperative axial computed tomography scan demonstrating a fibrous dysplasia of the left orbit including the optic canal. (B) Endoscopic endonasal uninarial approach. The Micro Claw is used to remove the optic canal roof. (C) Postoperative image demonstrating decompression of the optic canal. CN, cranial nerve; Op. Can., optic canal.

for paraclinoid aneurysms, ${ }^{8}$ for optic canal "unroofing" to treat parasellar tumors, ${ }^{10}$ to open the internal auditory canal in acoustic neuroma surgery, ${ }^{8}$ and for vertebral artery anterior decompression ${ }^{11}$ and spinal surgery. ${ }^{12-15}$ There are few reports in the literature regarding the application of the ultrasonic curette in endoscopic endonasal transsphenoidal and sinus surgery. ${ }^{4-7}$ However, to our knowledge, there is no previous report about the use of this equipment for extended applications of the endoscopic endonasal approach, in particular for the resection of the odontoid process, posterior clinoids, crista galli, and optic nerve decompression as well. During EES, a distinct advantage of the ultrasonic curette is the ability to work in a narrow corridor without the risk of circumferential tissue damage. The directionality of the Sonopet tip provides protection of tissues opposite the bone. Examples include removal of the posterior clinoid and crista galli, and optic nerve decompression. This situation is aggravated if the bone is mobile. For instance, when working at the craniovertebral junction, previous pathologic fractures or surgical manipulation can lead to a disconnection of the tip that may lead to a free bone fragment. During posterior clinoidectomy, this osseous process is often freely mobilized. Its removal is already challenging because of the proximity of the dura, pituitary gland, adjacent ICA, and oculomotor nerve. In 
such situations, a drill is ineffective and dangerous due to displacement of the fragment. The ultrasonic curette requires minimal pressure to remove the bone fragment without significant displacement.

The ultrasonic bone curette does not require a significant learning curve. However, we highlight that the ultrasonic curette by itself does not avoid the risk of injury to adjacent neurovascular structures. The most important factors to decrease the incidence of iatrogenic lesions are still the use of appropriate techniques and surgeon expertise.

Two limitations, economic considerations aside, of the ultrasonic bone curette were noticed. First, it is not adequate for heavy bone removal in wide, more superficial surgical areas (i.e., sphenoid sinus) because it is time consuming, less precise for fine end-on shaving, and does not provide any benefit when compared with high-speed drilling. ${ }^{7}$ As reported before, most of the bone removal in endonasal approaches is performed using high-speed drills and bone punches. ${ }^{7,16-23}$ We do not recommend the ultrasonic bone curette for routine bone removal due to a less aggressive tip when compared with coarse diamond or cutting drill bits, which could increase the procedure time significantly. In addition, removal, for instance, of the bone covering the parasellar or paraclival segments of the carotid is better performed with precise and meticulous high-speed drilling in combination with bone punches and dissectors. We reserve the ultrasonic bone curette for critical areas to decrease the risk of injury to adjacent soft tissue or neurovascular structures.

Second, the spray of the irrigation can hinder visualization for accurate and safe drilling, especially in narrow corridors. In such situations, an experienced surgical team (two surgeons using the four-hands technique) is imperative to maintain good visualization during the procedure.

\section{Conclusions}

The ultrasonic bone curette is a useful adjunct during EES of the skull base. It does not replace but rather complements the use of a high-speed drill. The mechanical characteristics of this surgical tool (nonrotational mechanism, low profile, directional cutting surface) provide protection to adjacent dura and neurovascular structures when working through narrow corridors. Another advantage of the ultrasonic curette is the resection of loose pieces of bone attached to the dura or ligaments. In this study, we demonstrated specific applications of the ultrasonic bone curette where it has advantages compared with the high-speed drill. Further studies are needed to prove the benefit of this surgical device, especially in decreasing the risk of injuries.

\section{Acknowledgment}

Matthew Tormenti, MD, was the originator of the concept of using the ultrasonic aspirator for removal of the inner cortical shell of the odontoid process.

The authors have no disclosure of funding, no financial support, no industry affiliations, and no personal or insti- tutional financial interest in the materials and devices described in this article.

\section{References}

1 Maroon JC. Skull base surgery: past, present, and future trends. Neurosurg Focus 2005;19(1):E1

2 Prevedello DM, Doglietto F, Jane JA Jr, Jagannathan J, Han J, Laws ER Jr. History of endoscopic skull base surgery: its evolution and current reality. J Neurosurg 2007;107(1):206-213

3 Snyderman CH, Pant H, Carrau RL, Prevedello D, Gardner P, Kassam $\mathrm{AB}$. What are the limits of endoscopic sinus surgery?: the expanded endonasal approach to the skull base Keio J Med 2009; 58(3):152-160

4 Yamasaki T, Moritake K, Nagai H, Uemura T, Shingu T, Matsumoto Y. A new, miniature ultrasonic surgical aspirator with a handpiece designed for transsphenoidal surgery. Technical note. J Neurosurg 2003;99(1):177-179

5 Pagella F, Giourgos G, Matti E, Colombo A, Carena P. Removal of a fronto-ethmoidal osteoma using the Sonopet omni ultrasonic bone curette: first impressions. Laryngoscope 2008;118(2): 307-309

6 Bruggers S, Sindwani R. Evolving trends in powered endoscopic sinus surgery. Otolaryngol Clin North Am 2009;42(5):789-798, viii

7 Cappabianca P, Cavallo LM, Esposito I, Barakat M, Esposito F. Bone removal with a new ultrasonic bone curette during endoscopic endonasal approach to the sellar-suprasellar area: technical note. Neurosurgery 2010;66(3, Suppl Operative):E118; discussion E118

8 Hadeishi H, Suzuki A, Yasui N, Satou Y. Anterior clinoidectomy and opening of the internal auditory canal using an ultrasonic bone curette. Neurosurgery 2003;52(4):867-870; discussion 870-871

9 Sawamura Y, Fukushima T, Terasaka S, Sugai T. Development of a handpiece and probes for a microsurgical ultrasonic aspirator: instrumentation and application. Neurosurgery 1999;45(5): 1192-1196; discussion 1197

10 Chang HS, Joko M, Song JS, Ito K, Inoue T, Nakagawa H. Ultrasonic bone curettage for optic canal unroofing and anterior clinoidectomy. Technical note. J Neurosurg 2006;104(4):621-624

11 Kim K, Isu T, Morimoto D, Kominami S, Kobayashi S, Teramoto A. Anterior vertebral artery decompression with an ultrasonic bone curette to treat bow hunter's syndrome. Acta Neurochir (Wien) 2008;150(3):301-303; discussion 303

12 Nakase H, Matsuda R, Shin Y, Park YS, Sakaki T. The use of ultrasonic bone curettes in spinal surgery. Acta Neurochir (Wien) 2006;148(2):207-212; discussion 212-213

13 Nakagawa H, Kim SD, Mizuno J, Ohara Y, Ito K. Technical advantages of an ultrasonic bone curette in spinal surgery. J Neurosurg Spine 2005;2(4):431-435

14 Kim K, Isu T, Matsumoto R, Isobe M, Kogure K. Surgical pitfalls of an ultrasonic bone curette (SONOPET) in spinal surgery. Neurosurgery 2006;59(4, Suppl 2):ONS390-ONS393; discussion ONS393

15 Ito K, Ishizaka S, Sasaki T, et al. Safe and minimally invasive laminoplastic laminotomy using an ultrasonic bone curette for spinal surgery: technical note. Surg Neurol 2009;72(5):470-475; discussion 475

16 de Divitiis E, Cappabianca P, Cavallo LM. Endoscopic transsphenoidal approach: adaptability of the procedure to different sellar lesions. Neurosurgery 2002;51(3):699-705; discussion 705-707

17 Cappabianca P, Cavallo LM, de Divitiis E. Endoscopic endonasal transsphenoidal surgery. Neurosurgery 2004;55(4):933-940; discussion 940-941

18 Kassam AB, Snyderman C, Gardner P, Carrau R, Spiro R. The expanded endonasal approach: a fully endoscopic transnasal approach and resection of the odontoid process: technical case report. Neurosurgery 2005;57(1, Suppl):E213; discussion E213 
19 Kassam A, Snyderman CH, Mintz A, Gardner P, Carrau RL. Expanded endonasal approach: the rostrocaudal axis. Part I. Crista galli to the sella turcica. Neurosurg Focus 2005;19(1):E3

20 Kassam A, Snyderman CH, Mintz A, Gardner P, Carrau RL. Expanded endonasal approach: the rostrocaudal axis. Part II. Posterior clinoids to the foramen magnum. Neurosurg Focus 2005;19(1):E4

21 Kassam AB, Gardner P, Snyderman C, Mintz A, Carrau R. Expanded endonasal approach: fully endoscopic, completely transnasal ap- proach to the middle third of the clivus, petrous bone, middle cranial fossa, and infratemporal fossa. Neurosurg Focus 2005; 19(1):E6

22 Laufer I, Anand VK, Schwartz TH. Endoscopic, endonasal extended transsphenoidal, transplanum transtuberculum approach for resection of suprasellar lesions. J Neurosurg 2007;106(3):400-406

23 Gardner PA, Kassam AB, Thomas A, et al. Endoscopic endonasal resection of anterior cranial base meningiomas. Neurosurgery 2008;63(1):36-52; discussion 52-54 